\title{
Kidney-pancreas transplant recipient with dysuria and hematuria
}

\author{
Abhilash Koratala $^{1}$ - Don H. Esprit ${ }^{1}$ - Alfonso H. Santos ${ }^{1} \cdot$ Karl L. Womer $^{1}$
}

Received: 22 August 2016/Accepted: 27 August 2016/Published online: 1 September 2016 (C) SIMI 2016

A 57 year-old male with combined kidney-pancreas transplant 10 years ago presented with suprapubic pain, dysuria, and hematuria that started 3 days prior to presentation. His pancreas is enteric-drained and the last C-peptide level was $2 \mathrm{ng} / \mathrm{mL}$ (normal: $0.9-4.3 \mathrm{ng} / \mathrm{mL}$ ). His maintenance immunosuppressive medications included tacrolimus $6 \mathrm{mg}$ twice a day, mycophenolate $750 \mathrm{mg}$ twice a day and prednisone $10 \mathrm{mg}$ daily. On examination, he was in apparent discomfort, afebrile, blood pressure $150 / 80 \mathrm{mmHg}$, pulse $88 \mathrm{bpm}$, otherwise unremarkable heart and lung findings, right lower quadrant allograft site was non-tender but the suprapubic area was distended and tender to palpation. Laboratory findings: white blood cell count was 9200 cells $/ \mathrm{mm}^{3}$ with $72.2 \%$ neutrophils, hemoglobin $11.5 \mathrm{~g} / \mathrm{dL}$, and serum creatinine was $1.2 \mathrm{mg} /$ $\mathrm{dL}$, blood glucose $98 \mathrm{mg} / \mathrm{dL}$. Urinalysis showed $8-10$ red blood cells/hpf, 20-25 white blood cells/hpf. Abdominal CT scan showed distended urinary bladder with abnormal urothelial enhancement and multiple foci of air in the bladder wall suggestive of Emphysematous Cystitis (Fig. 1a, b). The renal allograft was unremarkable, and the native kidneys were atrophic. Cystoscopy was performed which showed diffuse submucosal emphysematous lesions confirming the diagnosis of Emphysematous Cystitis
(Fig. 1c, d). Urine culture revealed $>100,000 \mathrm{CFU} / \mathrm{mL}$ of Klebsiella pneumoniae. Patient was treated with intravenous antibiotic therapy and recovered.

Emphysematous cystitis is a rare form of complicated urinary tract infection, whose characteristic feature is presence of gas within the bladder wall and lumen. Various gas-forming microbes including fungi have been implicated in emphysematous cystitis with Escherichia coli $(60 \%)$ and Klebsiella pneumoniae (10-20\%) being the two most common isolates [1]. The most common symptoms of emphysematous cystitis are abdominal pain and hematuria, occurring in about $80 \%$ of patients [2]. Though fever is more suggestive of pyelonephritis, it may be observed in approximately $30-50 \%$ of patients [1]. Pneumaturia (passage of gas in the urine) is a highly specific symptom, but is a rare patient complaint.

Diabetes mellitus, neurogenic bladder, bladder outlet obstruction, organ transplant and recurrent urinary tract infection are the major risk factors for this condition [3]. Among these risk factors, diabetes mellitus appears to be the strongest, and it is reported that almost $70 \%$ of patients with emphysematous cystitis are diabetics [1].

Computed tomography (CT scan) is the best imaging modality for the diagnosis and approximately $90 \%$ of the

Abhilash Koratala

abhilash.koratala@medicine.ufl.edu

1 Division of Nephrology, Hypertension and Renal

Transplantation, University of Florida,

P.O. Box 100224, Gainesville, FL 32610, USA 


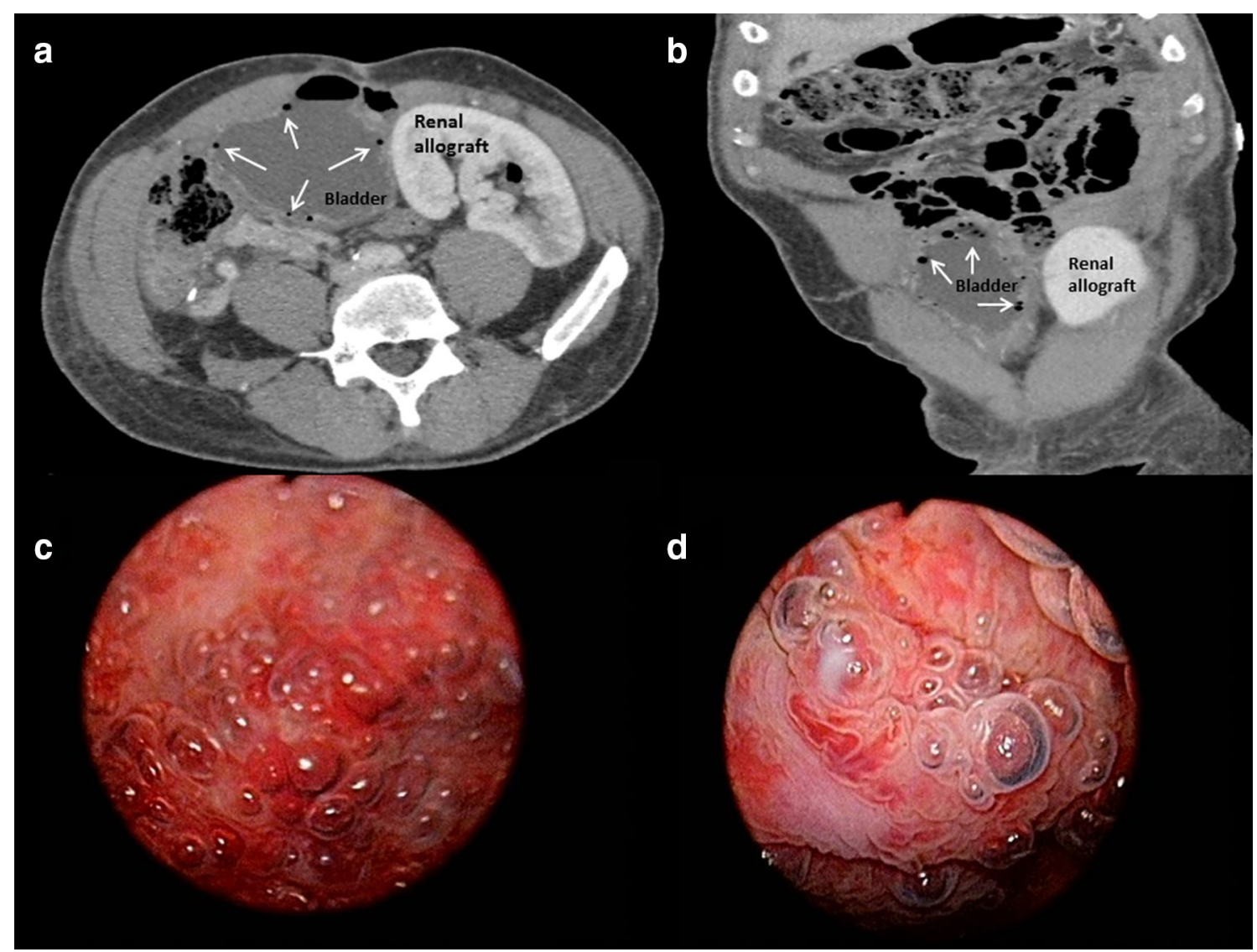

Fig. 1 a, b CT scan of the abdomen showing distended urinary bladder with abnormal urothelial enhancement and multiple foci of air in the bladder wall (arrows). c, d Cystoscopy images showing diffuse submucosal emphysematous lesions with erythematous bladder mucosa

cases are treated with medical therapy alone, while about $10 \%$ require combined surgical and medical intervention [4]. A delay in diagnosis or inappropriate treatment can result in bladder rupture, sepsis and death.

Acknowledgments Dr Gusto Gonzalez, MD, Urology.

\section{Compliance with ethical standards}

Conflict of interest The Authors declare that they have no conflict of interest.

Statement of human and animal rights All procedures followed were in accordance with the ethical standards of the responsible committee on human experimentation and with the Helsinki Declaration of 1975, as revised in 2013. No animals were used during the study.
Informed consent Informed consent was obtained from all individual participants included in the study.

\section{References}

1. Amano M, Shimizu T (2014) Emphysematous cystitis: a review of the literature. Int Med 53(2):79-82 (Epub 2012 Mar 1)

2. Grupper M, Kravtsov A, Potasman I (2007) Emphysematous cystitis: illustrative case report and review of the literature. Medicine (Baltimore) 86(1):47-53

3. Akalin E, Hyde C, Schmitt G, Kaufman J, Hamburger RJ (1996) Emphysematous cystitis and pyelitis in a diabetic renal transplant recipient. Transplantation 62(7):1024-1026

4. Thomas AA, Lane BR, Thomas AZ, Remer EM, Campbell SC, Shoskes DA (2007) Emphysematous cystitis: a review of 135 cases. BJU Int 100(1):17-20 (Epub 2007 May 17) 